\title{
Buruli Ulcer: Epidemiological, Clinical and Biological Profile of Patients in the Centre de Depistage et de Traitement d'Allada (Benin) from 2010 to 2014
}

\author{
Bérénice Dégboé1,2*, Christiane Koudoukpo ${ }^{3,4}$, Mouhaéminath Alimi1,2, \\ Ghislain Emmanuel Sopoh ${ }^{5,6}$, Fabrice Akpadjan ${ }^{1,2}$, Roch Christian Johnson ${ }^{7,8}$, \\ Nadège Agbéssi3 ${ }^{3,4}$, Hugues Adégbidi1,2, Félix Atadokpèdé1,2 \\ ${ }^{1}$ Faculté des Sciences de la Santé, Université d’Abomey-Calavi, Abomey-Calavi, Benin \\ ${ }^{2}$ Clinique universitaire de dermatologie-vénérologie, Centre National Hospitalier et Universitaire Hubert Koutoukou MAGA de \\ Cotonou, Cotonou, Benin \\ ${ }^{3}$ Faculté de Médecine de Parakou, Université de Parakou, Parakou, Benin \\ ${ }^{4}$ Clinique universitaire de dermatologie-vénérologie, Centre Hospitalier Universitaire Départemental du Borgou-Alibori, Parakou, Bénin \\ ${ }^{5}$ Institut Régional de Santé Publique, Université d’Abomey-Calavi, Ouidah, Benin \\ ${ }^{6}$ Centre de Dépistage et de traitement de l'ulcère de Buruli (CDTUB) d'Allada, Allada, Benin \\ ${ }^{7}$ Fondation Raoul FOLLEREAU, Paris, France \\ ${ }^{8}$ Centre Inter Facultaire de Formation et de Recherche en Environnement pour le Développement Durable, Université \\ d'Abomey-Calavi, Abomey-Calavi, Benin \\ Email: *kebdegboe@yahoo.fr
}

\begin{abstract}
How to cite this paper: Dégboé, B., Koudoukpo, C., Alimi, M., Sopoh, G.E., Akpadjan, F., Johnson, R.C., Agbéssi, N., Adégbidi, H. and Atadokpèdé, F. (2019) Buruli Ulcer: Epidemiological, Clinical and Biological Profile of Patients in the Centre de Depistage et de Traitement d'Allada (Benin) from 2010 to 2014. Journal of Cosmetics, Dermatological Sciences and Applications, 9, 67-82.

https://doi.org/10.4236/jcdsa.2019.92006
\end{abstract}

Received: February 14, 2019

Accepted: April 1, 2019

Published: April 4, 2019

\section{Copyright $\odot 2019$ by author(s) and} Scientific Research Publishing Inc. This work is licensed under the Creative Commons Attribution International License (CC BY 4.0).

http://creativecommons.org/licenses/by/4.0/

\begin{abstract}
Introduction: The objective of our work was to describe the epidemiological, clinical and biological profile of Buruli ulcer in "Centre de Dépistage et de traitement de l'ulcère de Buruli" (CDTUB) in Allada. Methods: A descriptive and retrospective study focused on new cases of Buruli ulcer received in the CDTUB of Allada from 2010 to 2014. The diagnosis of Buruli ulcer was based on epidemiological, clinical and biological arguments. Results: Over 5 years, 274 new cases of Buruli ulcer have been diagnosed. The average age of the patients was 12 years and the sex ratio was 0.8 . The average time to first consultation was 45 days. Clinically, $61 \%$ had a joint functional limitation. Lesions were ulcerated in $69 \%$ of cases, category I (26\%), category II (53\%), category III (21\%) and were present on the lower limbs in $57 \%$ of cases. Microscopy was positive in $65.7 \%$ of cases and PCR in $78.1 \%$ of cases. Microscopy supplemented by PCR confirmed the diagnosis in $81 \%$ of cases. Conclusion: The epidemiological, clinical and biological profile of Buruli ulcer in Allada was characterized by a predominant disease in children, a predominance of ulce-
\end{abstract}

Open Access 
rated forms and a decisive contribution of PCR to the diagnosis.

\section{Keywords}

Buruli Ulcer, Epidemiology, Clinical Profile, Biological Confirmation, Benin

\section{Introduction}

Buruli ulcer (BU) is an infectious disease caused by Mycobacterium ulcerans, clinically characterized by skin ulcers and histopathologically by necrotizing panniculitis [1] [2] [3] [4].

This pathology is one of the World Health Organization (WHO) 20 priority neglected tropical diseases, which prevail mainly in underdeveloped rural areas of Africa, Asia and South America [5]. It is the most common mycobacteriosis currently encountered after tuberculosis and before leprosy in a large number of countries in the intertropical zone [6]. Its epidemiology is closely linked to the aquatic ecosystem [1] [2].

BU mainly affects active children and adults considered to be the economic engine in these regions. It causes significant morbidity and functional disabilities and therefore constitutes a socio-demographic, economic and psychological threat [1] [2] [3] [4].

In response to the problem, the WHO launched a global Buruli ulcer initiative in Cote d'Ivoire in 1997. The first conference on Buruli ulcer followed in 1998 and resulted in the Yamoussoukro (Cote d'Ivoire) declaration [7] [8]. Benin subsequently organized a major conference in 2009 that yield to a second declaration called the "Cotonou Initiative" in order to improve the control of this disease through early detection and treatment [9].

This study aims to review the epidemiological, clinical and biological profile of Buruli ulcer patients received at the CDTUB in Allada since the launch of this initiative.

\section{Methods}

We conducted a retrospective, descriptive and analytical study that covered 5 years of activities from 2010 to 2014 at the "Centre de Dépistage et de traitement de l'ulcère de Buruli (CDTUB) d'Allada". Allada is a municipality located in southern Benin (Figure 1). This study extended to the seven decentralized centers in the municipalities of Sô-Ava, Calavi, Zè, Toffo, Allada, but attached to the CDTUB of Allada for the care of BU in the health zone. These centers are about 8 to 10 kilometers from the Allada's CDTUB. The CDTUB of Allada is the reference center for the management of BU in the Atlantic Department. It is one of the five most important centers established by the National Programme for the Control of BU (PNLUB).

We have identified all patients who have been managed in the Allada's CDTUB 


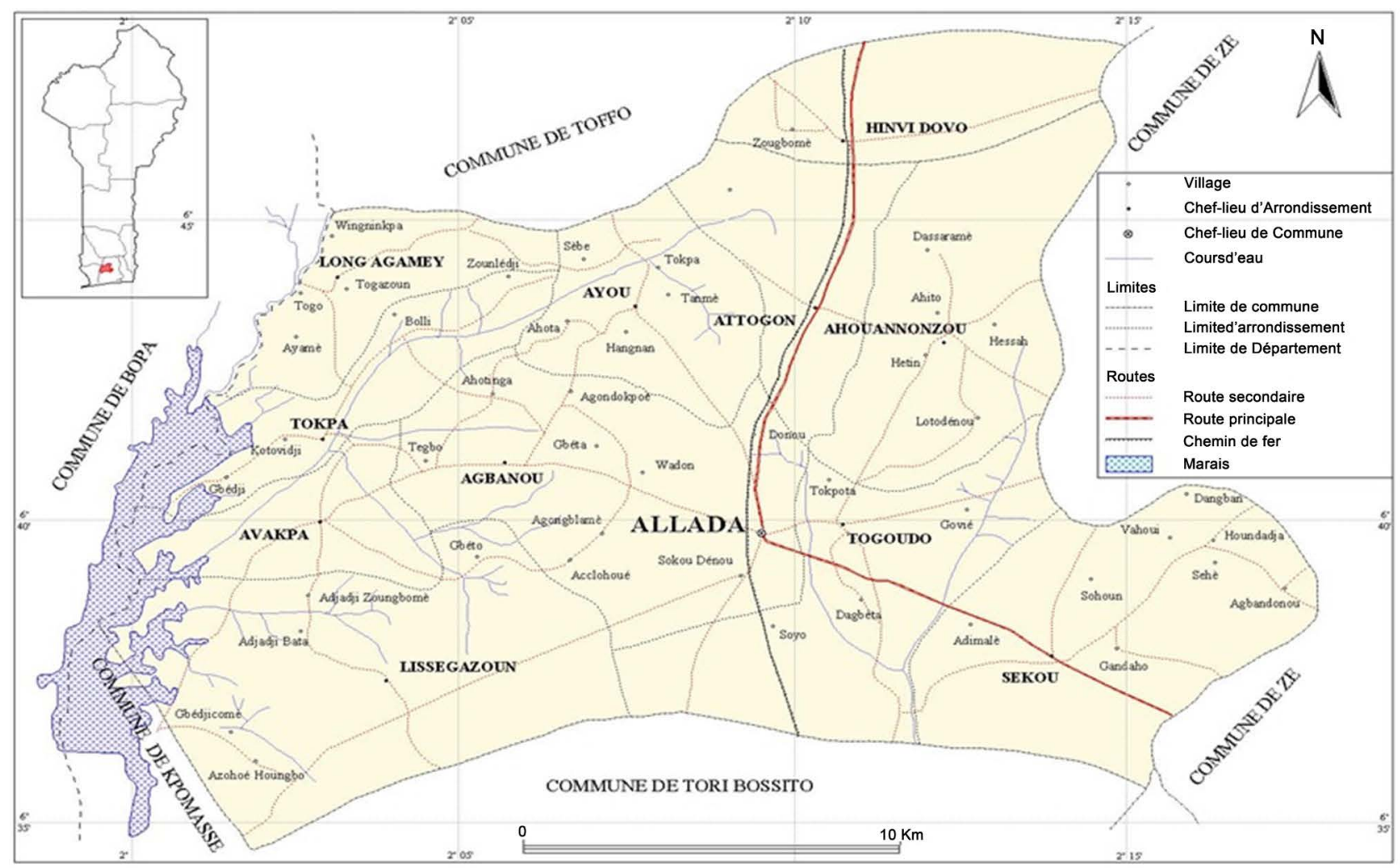

Figure 1. Geographic map of municipality of Allada.

and its associated centers on the basis of an exhaustive sampling. All patients diagnosed and treated whose records include complete information on the socio-demographic, clinical and biological characteristics of the patients were included. Patients who were not included were those with an inoperable record and those who were lost to follow up.

The diagnosis of BU was based on WHO epidemiological and clinical arguments [10] as well as biological results.

Clinical lesions have been described according to the WHO classification [10]. Category I is defined as a single small early lesion (nodule, papule, plaque and ulcer) less than $5 \mathrm{~cm}$ in diameter. Category II is single lesion (plaque and edema with or without ulceration, ulcerative lesions) between 5 and $15 \mathrm{~cm}$ in diameter. Category III covers all single lesions of more than $15 \mathrm{~cm}$ in diameter or multiple lesions, or any lesion located in a critical site (osteitis, bones, breasts, external genitalia, head, neck and face lesions).

Samples were collected for analysis based on the WHO guidelines for case confirmation [5]. The biological tests were microscopic examination on smears stained by the Ziehl-Neelsen method, thanks to Acid Fast Bacilli (AFB), performed in the laboratory of the CDTUB of Allada and the polymerase chain reaction (PCR) performed in the Reference Laboratory of Mycobacteria (LRM) of Cotonou, on swabbing of undermined edges of ulcers, or fine needle aspiration on non-ulcerative lesions as recommended by WHO [5].

The data were collected using a survey sheet. They were then coded and en- 
tered anonymously with Excel 2007 software. The statistical analysis was performed with the same software using descriptive and analytical epidemiological techniques. The chi2 test was used for comparisons with a threshold $\mathrm{p} \leq 0.05$ considered significant.

Access to the registry was approved by the institutional review board of the Allada's CDTUB and the PNLLUB's (National Buruli ulcer control) authorities.

\section{Results}

A total of 327 cases of $\mathrm{BU}$ were diagnosed, resulting in a detection rate of 65 cases/year. Fifty-three of these patients were not included in the study because they were lost to follow up. So, we included 274 patients.

The median age of the patients in our study was 12 years with extremes of 1 year and 80 years. A breakdown by age group (Table 1) shows that children under 15 years of age constituted the majority of cases, $57.7 \%$. The sex ratio was 0.8 , giving a slight female predominance.

Two hundred and ten of the 274 patients (76.6\%) lived in an environment where there was frequent proximity or contact with a watercourse. Thirty patients $(10.9 \%)$ of this population went to swamps or rivers for their water supply.

Almost all patients, two hundred and forty-eight (90.5\%) had a low level of education, of which $53.3 \%$ were not in the school system and $37.2 \%$ reached the primary level.

The median time to consultation was 45 days with extremes of 7 days and 1825 days.

One hundred and ninety-seven patients $(76.6 \%)$ received phytotherapy care before the consultation.

Only one patient was HIV-positive.

One hundred and sixty-seven patients (61\%) already had a functional limitation before the consultation.

The morphological aspect of the lesions consisted of ulcerated lesions (69\%; Figure 2), plaque lesions (20.4\%; Figure 3), associated lesions (5.5\%), nodules and oedemas (2.5\%; Figure 4 and Figure 5) each (Figure 6). According to the WHO classification, the majority, 144 (52.5\% had category II lesions, 72 (26.3\%) had category I lesions and 58 (21.2\%) had category III lesions.

The majority of lesions were localized to limbs (91.6\%) with a predominance to lower limbs (57\%) as shown in Figure 7.

Table 1. Age (years) distribution of the 274 patients followed for Buruli ulcer in the CDTUB of Allada from 2010 to 2014.

\begin{tabular}{ccc}
\hline & Number & Frequency (\%) \\
\hline$<15$ & 158 & 57.7 \\
$15-40$ & 74 & 27 \\
$>40$ & 42 & 15.3 \\
Total & 274 & 100 \\
\hline
\end{tabular}




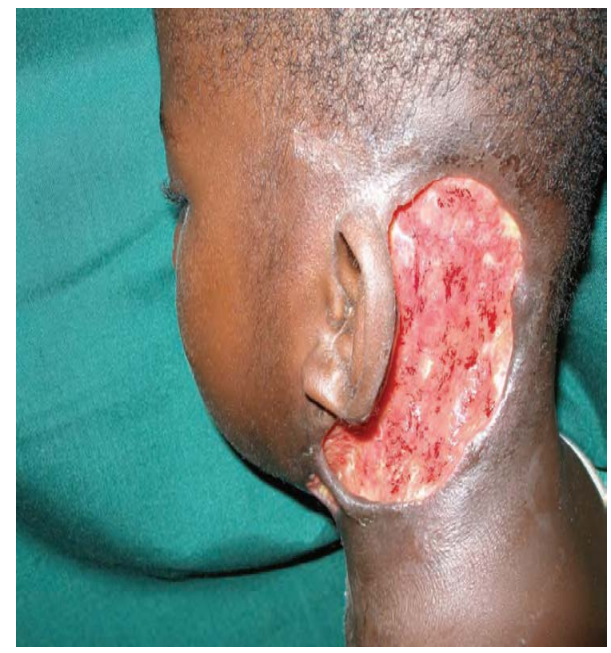

Figure 2. Ulcerative lesion of Buruli ulcer on retro-auricular region.

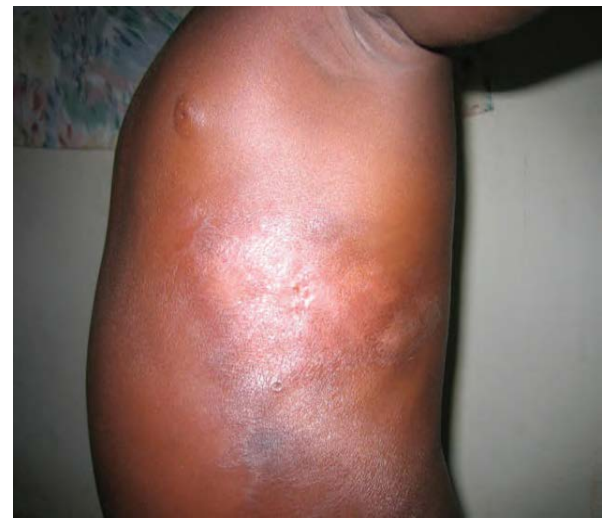

Figure 3. Plaque of Buruli ulcer on the trunk.

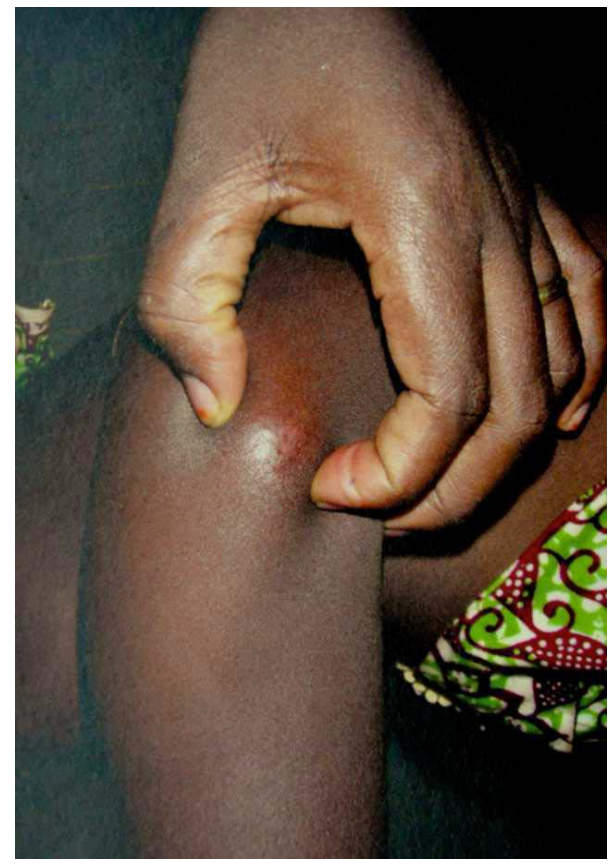

Figure 4. Nodular lesion of Buruli ulcer on lower limb. 


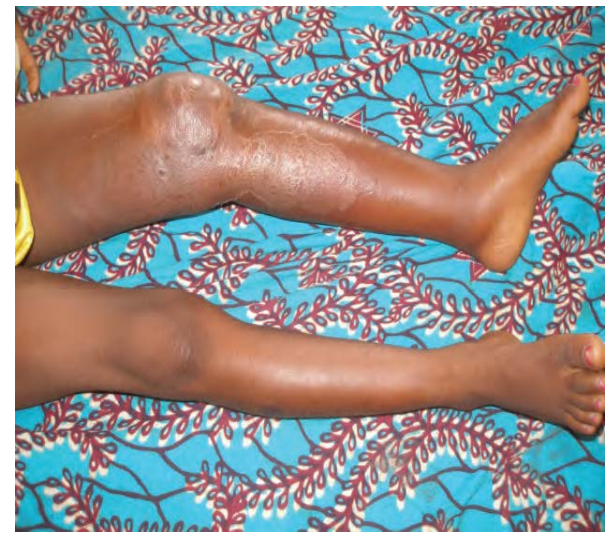

Figure 5. Oedema lesion of Buruli ulcer on left lower limb.

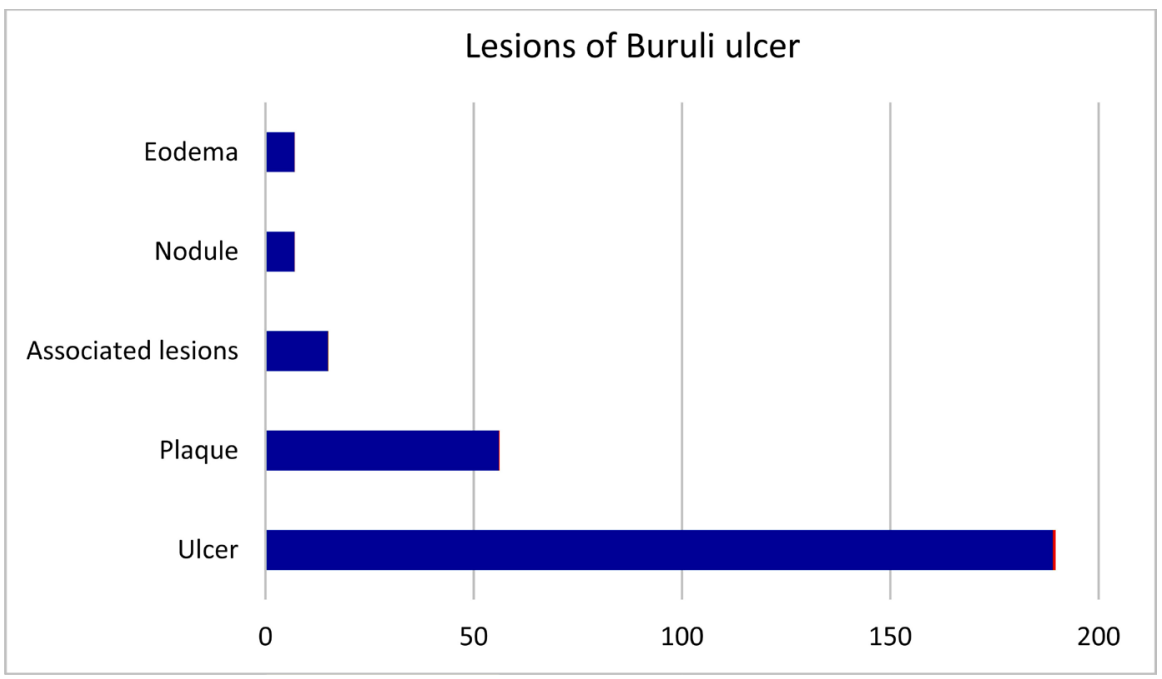

Figure 6. Distribution by morphological lesions of the 274 patients followed for Buruli ulcer in the CDTUB of Allada from 2010 to 2014.

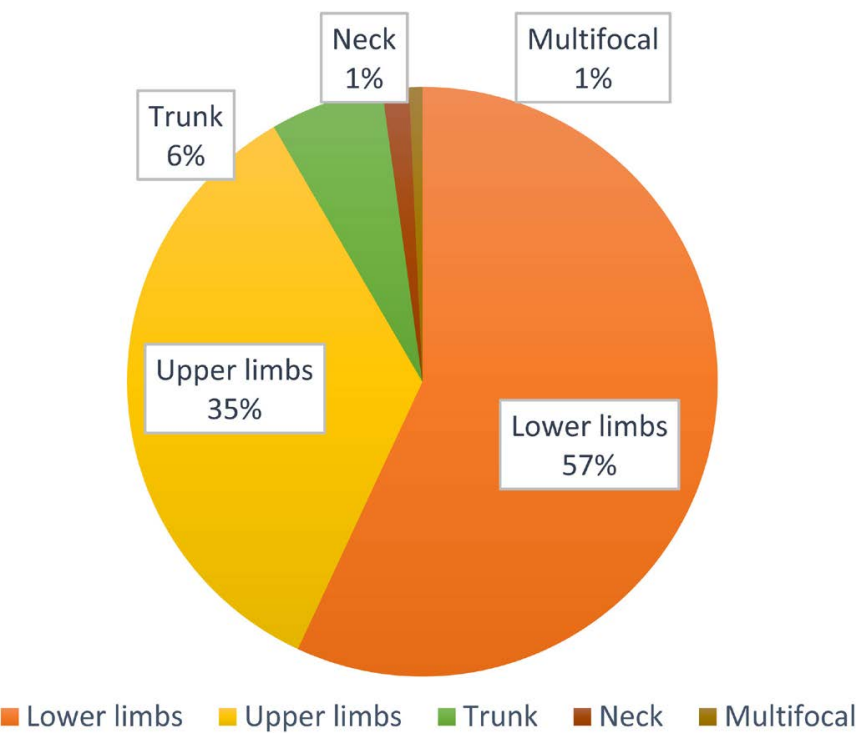

Figure 7. Distribution by sites of lesions of the 274 patients followed for Buruli ulcer in the CDTUB of Allada from 2010 to 2014. 
Microscopy was positive in 180 patients (65.7\%) and negative in 94 (34.3\%) patients. Microscopy was consistently positive in nodular lesions (100\%), followed by associated lesions in $93.3 \%$ of cases and oedema in $85.7 \%(p=0.02)$, while it was less positive in ulcerated (63.5\%) and plaque (59\%) lesions. The proportion of positive microscopy specimens was higher in category II lesions, at $72.2 \%$ and category III lesions, at $62.1 \%(\mathrm{p}=0.04)$ compared to $55.6 \%$ for category I lesions. The proportion of positive samples taken by microscopy was higher for neck (75\%) and trunk (70.6\%) lesions compared to lower limbs $(67.3 \%)$ and upper limbs (62.1\%) lesions.

PCR was positive in 214 patients (78.1\%) and negative in the remaining 60 (21.9\%) patients. Of the 94 patients with negative microscopy, 42 (44.7\%) had a positive PCR (Table 2). PCR was negative in 8 (4.4\%) patients with positive microscopy. Using both positive microscopy and PCR, the diagnosis of BU was confirmed in 222 patients (81\%); (214 positive PCR +8 positive microscopies with negative PCR). The proportion of positive samples taken by PCR was higher for trunk (82.3\%) and upper limbs (81.9\%) lesions compared to lower limbs (75.8\%) and neck (75\%) lesions.

\section{Discussion}

The detection rate of BU in the Allada's CDTUB from 2010 to 2014 was 65 cases/year. This result is significantly higher than that observed in Ghana in 2016 [3] and Australia [4] in 2011 where an incidence of 35 cases/year and 12 cases/year was reported. On the other hand, another study carried out in another CDTUB in Pobè [11] found a higher incidence of 176 cases/year. A literature review showed that $\mathrm{BU}$ is characterized by a high incidence in endemic countries in West and Central Africa such as Benin, Ivory Coast, Ghana, Cameroon, Democratic Republic of Congo [1] [2] [12] [13]. In Benin, the areas of high endemicity are mainly in the south of Benin, in the region surrounding the Lama depression between the Couffo and Ouémé rivers. In 2011, these areas were Lalo, Ounhi, Zè, Adjohoun, Bonou, Dangbo with a prevalence between 54 and 101 per 1000 inhabitants [14] [15]. But overall, there has been a significant decrease in the incidence of BU, from 1195 new cases in 2006 to 311 new cases in 2015. And the health zone covered by the Allada CDTUB is no longer a highly endemic zone, as our results between 2010 and 2014 show [14] [16].

A study in Guyana showed a decrease in incidence over time from an average of 6.07 cases/year in 1969 to 3.49 cases/year in 2013. These authors concluded

Table 2. Distribution of PCR results according to microscopy results in the 274 patients followed for Buruli ulcer in the CDTUB of Allada from 2010 to 2014.

\begin{tabular}{cccc}
\hline & Positive PCR & Negative PCR & Total \\
\hline Positive microscopy & $172(62.8 \%)$ & $8(2.9 \%)$ & $180(65.7 \%)$ \\
Negative microscopy & $42(15.3 \%)$ & $52(19 \%)$ & $94(34.3 \%)$ \\
Total & $214(78.1 \%)$ & $60(21.9 \%)$ & $274(100 \%)$ \\
\hline
\end{tabular}


that there was a change in the epidemiological profile of BU in Guyana from a West African profile with a high incidence to an Australian profile with a low incidence. This decrease in incidence, which remains the main objective of the WHO, is due to an improvement in living conditions and hygiene observed over time in Guyana [17]. We believe that improving hygiene and health conditions would help, as in Guyana, to reduce the incidence of BU in these areas of high prevalence.

BU in Allada's CDTUB was a childhood disease over the study period. This is confirmed because children under 15 years of age constituted the majority of cases (57.7\%). This is the age group reported as the most frequent in most studies conducted in endemic countries of West and Central Africa [3] [18]-[23]. This child dominance could be explained by several hypotheses of which the vulnerability of this age group due to a still immature immune system. This fact is reinforced on the one hand by insufficient coverage of BCG ("Bacille Calmette Guérin") vaccination among children in these regions. On the other hand, by the increased frequency of intestinal parasitosis, which also remains endemic in these regions and particularly in children. These parasitoses are thought to reduce the effectiveness of immune responses against mycobacteria [1] [2] [12]. The more frequent exposure of children with unprotected clothing during work or entertainment activities around water points could also contribute to this high infant prevalence.

In contrast in Australia, children under 15 years of age represent only $8 \%$ while the predominant age group is that of patients over 60 years of age, or $52 \%$. The authors do not link this high prevalence to more frequent exposure to $\mathrm{My}$ cobacterium ulcerans, but rather to a decrease in the protective power of BCG vaccination with age [4].

Children under 15 years of age were the predominant layer in Guyana until 1984 with a prevalence of more than $70 \%$ to gradually decrease and reach $21 \%$ in 2013 to make way for the 50 and over age group [17]. All these observations lead us to wonder if young age is really a risk factor for BU as found in several studies. Through the age dichotomy between developed and less developed countries, as well as the age transition observed in Guyana, we would rather suggest a link between socio-economic and environmental conditions and transmission patterns. This could be one of the important keys to combating BU in underdeveloped endemic countries through improved living conditions, hygiene and vaccination coverage.

A slight female predominance was reported in our study with a sex-ratio of 0.8 . The results of previous studies in this regard are contradictory [1] [2] [3] [4] [11] [19] [23]-[29]. This leads us to conclude that, regardless of the region, gender is not a risk factor for BU. It is mainly the socio-economic and cultural conditions that, according to gender, determine the different activities that expose people to infection by Mycobacterium ulcerans.

The majority of patients in our study (76.6\%) lived in an environment where there was frequent contact with a watercourse. Previous studies have shown that 
the prevalence of $\mathrm{BU}$ is higher near stagnant water points or aquatic reservoirs, particularly in less developed endemic countries. An infection favoured by insects (mosquitoes) is sometimes suspected. In addition, proximity or contact with a water source is a consistently reported risk factor in most of these studies [1] [2] [5] [12] [13] [17] [18] [19] [20] [23] [28] [30]. Access to safe domestic water source should be essential for effective control in endemic underdeveloped countries. Adequate access to WASH (Water, Sanitation and Hygiene) services is therefore a challenge for both healthy and non-healthy populations in many countries and communities with high levels of BU [31] [32] [33] [34].

In our study population $53.3 \%$ of patients were uneducated. Our result is consistent with those found in an endemic area in southern Ivory-Coast and in a population of gold miners in Ghana, which is $56.9 \%$ and $52 \%$ of uneducated patients [19] [35]. A lack of education does not appear to be a direct risk factor for $\mathrm{BU}$ according to a meta-analysis of studies conducted in 14 endemic regions [1]. Rather, it would condition the behaviours and practices that expose people to infection with Mycobacterium ulcerans [1] [17]. On the other hand, uneducated patients most often consult late, which exposes them to delayed care and more serious complications.

Due to patients' poor access to health services in remote rural areas, they often present at a late stage of the disease. The median time to consultation in our study was 45 days, but this time was highly variable with extremes of 7 days and 1825 days. This average is identical to that found by the Australian team, which is 42 days [4]. Capela et al. in the same center reported an average delay of 101.1 days between 2005 and 2013 [24]. Allodé et al. in the CDTUB of Zagnanado in Benin from 2006 to 2008 [28] reported an average consultation time of 372 days with extreme values of 7 days and 7300 days. This reduction in consultation time observed in our study could be explained by a better knowledge of the disease by the population over time. However, Capela et al. showed that this time varies with the severity of the lesions, ranging from an average of 32.5 days for pre-ulcerative lesions to 60 days for ulcers and category III lesions, 90 days for associated lesions and 365 days for bones [24]. We can therefore conclude that a long consultation period exposes to severe forms of the disease. This could jeopardize healing at the cost of heavy therapeutic measures and disabling sequelae. Awareness-raising is therefore essential to educate the population about the first signs of BU.

More than three-quarters of patients used herbal to treat their lesions before the consultation. The same observation has been made in most endemic countries of the sub-region where patients between $41 \%$ and $90 \%$ use herbal as a first-line treatment [19] [28] [35] [36]. This practice undeniably extends the consultation period, which has the consequences listed above. This phytotherapy, often used under dubious conditions, could also be the cause of the superinfections observed [37]. Collaboration with traditional healers could be considered in addition to raising public awareness to refer cases early on. 
The patients did not have a specific medical history in our series; only one patient was HIV-positive. HIV seroprevalence of $2 \%$ has been reported in Cameroon, 2.6\% in Benin and 5.2\% in Ghana [38] [39] [40]. According to the WHO, HIV infection is not a risk factor but, because it weakens the immune system, it makes Mycobacterium ulcerans infection more aggressive. Co-infected patients often have multifocal lesions and bones [10] [41]. Research is therefore needed to determine whether or not individual factors of susceptibility to BU exist. In addition, the management of BU/HIV co-infection may require the application of the guidelines as for the management of tuberculosis/HIV co-infection [42].

In the absence of pain, the appearance of the ulcer is the most characteristic stage of BU that leads patients to consult. In our study, there was a clear predominance of ulcerous lesions; $69 \%$ versus $25.4 \%$ of pre-ulcerative lesions. The majority of previous studies, even in developed countries, report a prevalence well above 65\% [3] [4] [11] [17] [19] [20] [25] [27] [29] [43]. This percentage must decrease to $60 \%$ in each district or country according to the WHO objective because ulcerative lesions require heavier therapeutic measures and more frequently lead to disabling sequelae [5]. It is therefore urgent to establish effective awareness on the recognition of pre-ulcerative lesions.

The WHO recommends that cases of functional limitation be reduced to less than $15 \%$ before the consultation [5]. Allada's CDTUB over the study period is still very far from this objective since $61 \%$ of patients already had a functional limitation before the consultation. Our result is similar to that reported in Nigeria by Ukwaja et al. in 2013, which was 67\% [20]. The latter links this high proportion of functional impotence to the high prevalence of category III lesions. However, the frequency of category III lesions in our series is significantly lower compared to that of Nigerians, at $21.2 \%$ compared to $52.8 \%$. Allodé in the CDTUB of Zagnanado in Benin, Phanzu and Obvala in Congo and Porten in Cameroon have identified functional limitations in less than one-third of their patients, despite the high proportion of ulcers and category III lesions [28] [44] [45] [46]. In contrast, Yotsu in Japan from 2008 to 2014 [46] had not seen any cases of limitation at the time of diagnosis. The paradox that emerges from these results leads us to suggest the existence of other factors than time delay before consultation that determine the severity of the infection. We can mention among other things the virulence of the different strains of Mycobacterium ulcerans according to areas, individual genetic factors as well as local immune responses.

Among our patients, $21.2 \%$ had category III lesions. Very few centers [35] [47] [48] have been able to achieve the WHO objectives in this sense, which recommends having less than $25 \%$ of category III lesions in each country at the last meeting in 2015 [5]. This result is encouraging compared to those reported in previous studies in Benin where the proportion of lesions in this category varies between 29.8\% and 36.3\% [11] [24] [47] [49]. On the other hand, Couppié in French Guyana in 2010 [26], Nakanaga in 2009 and Yotsu in 2014 in Japan [26] [50] did not note any category III lesions. Beyond the WHO target, endemic 
countries must increase their efforts to significantly reduce category III and category II lesions at the time of diagnosis.

In almost all cases (91.6\%), lesions were localized to limbs with a predominance to lower limbs (57\%). Regardless of the endemic regions, these locations are the most frequent with preferential damage to the lower limbs observed in more than 50\% of cases [1] [3] [4] [11] [17] [19] [20] [24] [25] [29] [51]. These observations could be explained by the fact that the exposed parts of the body are directly exposed to the potential vector of the disease. They thus provide some insight into the understanding of how the disease is transmitted.

In our study, microscopy was performed in conjunction with PCR for all patients. This method confirmed the diagnosis of BU in $81 \%$ of patients compared to $78.1 \%$ for PCR alone and $65.7 \%$ for microscopy alone. WHO recommends the use of at least two bioassays to confirm the diagnosis of BU [1].

Our positivity rate for Ziehl-Neelsen staining microscopy is higher than that of most of the previous studies, which reported a positivity between $22.3 \%$ and 56\% [3] [11] [17] [20] [24] [25] [44] [52] [53]. Avokan in 2011 [54] and Adè in 2012 [23] in Benin had noted rates of $33.6 \%$ and $38 \%$ respectively using auramine staining. In addition, the positivity of microscopy in our series is statistically higher for nodular, associated and edematous lesions on the one hand, and category II and III lesions on the other. It seems to be more important for neck and trunk lesions. These differences could therefore be related to differences in methodological approaches, the type of lesion and perhaps the sampling areas, among other things.

Our positivity rate of Mycobacterium ulcerans DNA PCR testing is consistent with those reported by the majority of authors in the literature [11] [23] [25] [48] [53] [55] [56] [57]. However, some studies have found lower rates between $32.8 \%$ and $63.2 \%$ [3] [29] [58]. On the other hand, higher rates have been reported in previous studies conducted in the same center between 2005 and 2013, in Nigeria and Guyana ranging from $87.8 \%$ to $90.3 \%$ [17] [20] [24]. The reasons given for these variabilities could be the same as for microscopy.

Other methods must be developed to diagnose pre-ulcerative lesions as early as possible, especially as they are less specific and less suggestive of Mycobacterium ulcerans infection. Alternative methods such as serology and chromatography are under evaluation. Serological detection of antibodies against $\mathrm{Myco}$ bacterium ulcerans specific antigens has shown mixed results. In addition to detecting patients with BU, the tests were positive in healthy carriers. Thin layer fluorescent chromatography highlighting mycolactone is used for a variety of samples taken from lesions. It has shown a sensitivity of $73 \%$ and a specificity of $86 \%$; however, this method is only in the early stages of its performance assessment to be retained as a diagnostic tool [1].

\section{Conclusions}

In addition to its high detection rate in the CDTUB of Allada, BU remains a public 
health problem because it frequently affects children to limbs in a context of functional impotence.

The low level of education of the population and the use of herbal as a first-line treatment were leading to a delayed diagnosis, so that patients often present at the ulcer stage.

The microscopy completed by PCR makes it possible to confirm the majority of cases.

Improving living conditions and hygiene, as well as raising awareness and educating the population, are also important pillars for the control of this endemic disease.

\section{Conflicts of Interest}

The authors declare no conflicts of interest regarding the publication of this paper.

\section{References}

[1] Guarner, J. (2018) Buruli Ulcer: Review of a Neglected Skin Mycobacterial Disease. Journal of Clinical Microbiology, 56, e01507-e01517.

[2] Jacobsen, K.H. and Padgett, J.J. (2010) Risk Factors for Mycobacterium ulcerans Infection. International Journal of Infectious Diseases, 14, e677-e681. https://doi.org/10.1016/j.ijid.2009.11.013

[3] Yeboah-Manu, D., Aboagye, S.Y., Asare, P., Asante-Poku, A., Ampah, K., Danso, E., et al. (2018) Laboratory Confirmation of Buruli Ulcer Cases in Ghana, 2008-2016. PLoS Neglected Tropical Diseases, 12, e0006560. https://doi.org/10.1371/journal.pntd.0006560

[4] Boyd, S.C., Athan, E., Deborah Friedman, N., Hughes, A., Walton, A., Callan, P., McDonald, A. and O'Brien, D.P. (2012) Epidemiology, Clinical Features and Diagnosis of Mycobacterium ulcerans in an Australian Population. Medical Journal of Australia, 196, 341-344. https://doi.org/10.5694/mja12.10087

[5] OMS (2015) Ulcère de Buruli-Infection à Mycobacterium ulcerans. Aide-mémoire N0. 199. http://www.who.int/mediacentre/factsheets/fs199/fr/

[6] Aubry, P. (2013) Ulcère de Buruli. Actualités 2013, Diplôme de médecine tropicale des pays de l'Océan Indien, $5 \mathrm{p}$.

[7] Kanga, J.-M., Kacou, E.D., Kouame, K., Kassi, E., Kaloga, M., Yao, J.K., et al. (2004) Buruli Ulcer: Epidemiological, Clinical and Therapeutic Aspects in the Ivory Coast. MedTrop, 64, 238-242.

[8] Bourée, P. (2012) Une pathologie tropicale en expansion: L'ulcère de Buruli. Antibiotiques, 12, 55-60. https://doi.org/10.1016/j.antib.2009.09.007

[9] Réunions sur l'ulcère de Buruli à Cotonou (2009) Bénin. www.who.int/buruli/events/BU_Summary_report_Cotonou_fr.pdf

[10] OMS (2012) Traitement de l'infection à Mycobacterium ulcerans (ulcère de Buruli). Recommandations à l'intention des agents de santé, $67 \mathrm{p}$.

[11] Vincent, Q.B., Ardant, M.-F., Adeye, A., Goundote, A., Saint-André, J.-P., Cottin, J., et al. (2014) Clinical Epidemiology of Laboratory-Confirmed Buruli Ulcer in Benin: A Cohort Study. Lancet Global Health, 2, e422-e430. 
https://doi.org/10.1016/S2214-109X(14)70223-2

[12] O’Brien, D.P. (2017) Unlocking of the Secrets of Mycobacterium ulcerans Disease Transmission. Lancet Planet Health, 1, e52-e53. https://doi.org/10.1016/S2542-5196(17)30026-8

[13] Van der Werf, T.S., Stienstra, Y., Johnson, R.C., Phillips, R., Adjei, O. and Fleischer, B. (2005) Mycobacterium ulcerans Disease. Public Health Reviews, 83, 785-791.

[14] Johnson, R.C., Sopoh, G.E., Barogui, Y., Dossou, A., Fourn, L. and Zohoun, T. (2008) Mise en place d'un système de surveillance de l'ulcère de Buruli au Bénin: point de 4 années de surveillance. Cahiers Santé, 18, 9-13.

[15] Sopoh, G.E., Johnson, R.C., Anagonou, S.Y., Barogui, Y.T., Dossou, A.D., Houézo, J.G., et al. (2011) Buruli Ulcer Prevalence and Altitude, Benin. Emerging Infectious Diseases, 17, 153-154. https://doi.org/10.3201/eid1701.100644

[16] Programme national de lutte contre les maladies transmissibles-République du Bénin (2017) Plan directeur national de lutte intégrée contre les maladies tropicales négligées 2016-2020.

[17] Douine, M., Gozlan, R., Nacher, M., Dufour, J., Reynaud, Y., Elguero, E., et al. (2017) Mycobacterium ulcerans Infection (Buruli Ulcer) in French Guiana, South America, 1969-2013: An Epidemiological Study. Lancet Planet Health, 1, e65-e73. https://doi.org/10.1016/S2542-5196(17)30009-8

[18] Debacker, M., Portaels, F., Aguiar, J., Steunou, C., Zinsou, C., Meyers, W. and Dramaix, M. (2006) Risk Factors for Buruli Ulcer, Benin. Emerging Infectious Diseases, 12, 1325-1331. https://doi.org/10.3201/eid1209.050598

[19] N’krumah, R.T.A.S., Koné, B., Tiembre, I., Cissé, G., Pluschke, G., Tanner, M., et al. (2016) Socio-Environmental Factors Associated with the Risk of Contracting Buruli Ulcer in Tiassalé, South Côte d'Ivoire: A Case-Control Study. PLoS Neglected Tropical Diseases, 10, e0004327. https://doi.org/10.1371/journal.pntd.0004327

[20] Ukwaja, K.N., Meka, A.O., Chukwuka, A., Kingsley, B., et al. (2016) Buruli Ulcer in Nigeria: Results of a Pilot Case Study in Three Rural Districts. Infectious Diseases of Poverty, 5, 39. https://doi.org/10.1186/s40249-016-0119-8

[21] Maman, I., Tchacondo, T., Banla Kere, A., Piten, E., Beissner, M., Kobara, Y., et al. (2018) Risk Factors for Mycobacterium ulcerans Infection (Buruli Ulcer) in Togo: A Case-Control Study in Zio and Yoto Districts of the Maritime Region. BMC Infectious Diseases, 18, 48. https://doi.org/10.1186/s12879-018-2958-3

[22] Bingidimi, S. (2015) Évaluation du protocole de l'OMS sur le traitement de l'ulcère de Buruli à l'hôpital général de référence IME/Kimpese. WHO Buruli Ulcer Meeting, Geneva, Switzerland.

[23] Adè, N. (2012) Évaluation de la PCR en temps réel pour le diagnostic de l'ulcère de Buruli. Thèse de pharmacie No. 074, Faculté des Sciences de la Santé, Université d'Abomey-Calavi, $93 \mathrm{p}$.

[24] Capela, C., Sopoh, G.E., Houezo, J.G., Fiodessihoué, R., Dossou, A.D., Costa, P., et al. (2015) Clinical Epidemiology of Buruli Ulcer from Benin (2005-2013): Effect of Time-Delay to Diagnosis on Clinical Forms and Severe Phenotypes. PLoS Neglected Tropical Diseases, 9, e0004005. https://doi.org/10.1371/journal.pntd.0004005

[25] Couppié, P. (2011) L'infection à Mycobacterium ulcerans (Ulcère de Buruli) en Guyane Française en 2010. Réunion annuelle de l'OMS sur l'ulcère de Buruli, Genève, Suisse.

[26] Nakanaga, K., Hoshino, Y. and Ishii, N. (2010) Ulcère de Buruli au Japon: 14 cas d'infection à Mycobacterium ulcerans, sous-espèce shinshuense de 1980 à 2009. 
Réunion annuelle de l'OMS sur l'ulcère de Buruli, Genève, Suisse.

[27] Herbinger, K.H., Nitschke J., Helfrich, K., Fleischmann, E., Beissner, M., Ebekalisai, P., et al. (2010) Rapport sur la confirmation des cas d'UB par les laboratoires au Togo. Réunion annuelle de l'OMS sur l'ulcère de Buruli, Genève, Suisse.

[28] Allode, S.A., Hodonou, M.A., Gbessi, G., Mensah, E.A.D., Hans-Moevi, A., Olory-Togbé, J.L., et al. (2013) Aspects épidémiologiques et cliniques de l'ulcère de Buruli dans un centre de dépistage et de traitement au Bénin. A propos de trois années d'activités. Médecine d' Afrique Noire Electronique, 60, 139-144.

[29] Obvala, D. (2011) Situation épidémiologique de l'ulcère de Buruli en 2010 au Congo en 2010. Réunion annuelle de l'OMS sur l'ulcère de Buruli, Genève, Suisse.

[30] Christinet, V., Di Benedetto, C., Comte, E. and Calmy, A. (2012) Buruli et VIH: une interaction complexe et peu étudiée. Revue Medicale Suisse, 8, 1792-1793.

[31] Williamson, H.R., Benbow, M.E., Campbell, L.P., Johnson, C.R., Sopoh, G., et al. (2012) Detection of Mycobacterium ulcerans in the Environment Predicts Prevalence of Buruli Ulcer in Benin. PLoS Neglected Tropical Diseases, 6, e1506. https://doi.org/10.1371/journal.pntd.0001506

[32] Johnson, R.C., Boni, G., Barogui, Y., Sopoh, G.E., Houndonougbo, M. and Anagonou, E. (2015) Assessment of Water, Sanitation, and Hygiene Practices and Associated Factors in a Buruli Ulcer Endemic District in Benin (West Africa). BMC Public Health, 15, 801. https://doi.org/10.1186/s12889-015-2154-y

[33] Johnson, R.C., Makoutodé, M., Sopoh, G.E., Elsen, P., Gbovi, J., Pouteau, L.H., et al. (2005) Distribution in Benin. Emerging Infectious Diseases, 11, 500-501. https://doi.org/10.3201/eid1103.040597

[34] Nackers, F., Johnson, R.C., Glynn, J.R., Zinsou, C., Tonglet, R. and Portaels, F. (2007) Environmental and Health-Related Risk Factors for Mycobacterium ulcerans Disease (Buruli Ulcer) in Benin. American Journal of Tropical Medicine and $\mathrm{Hy}$ giene, 77, 834-836. https://doi.org/10.4269/ajtmh.2007.77.834

[35] Nzilanye Iddrisah, F., Yeboah-Manu, D., Nortey, P.A., Nyarko, K.M., et al. (2016) Exposure of Small-Scale Gold Miners in Prestea to Mercury, Ghana, 2012. Pan African Medical Journal, 25, 13.

[36] Barthelmé, B., Stoffel, V., Chagué, F., Jacquenet, F. and Lachambre, A. (2001) Ulcères de Buruli: 14 cas en 5 semaines dans une sous-préfecture rurale du Bénin. Press Medya, 30, 1071.

[37] Barogui, Y.T., Klis, S., Bankolé, H.S., Sopoh, G.E., Mamo, S., et al. (2013) Towards Rational Use of Antibiotics for Suspected Secondary Infections in Buruli Ulcer Patients. PLoS Neglected Tropical Diseases, 7, e2010. https://doi.org/10.1371/journal.pntd.0002010

[38] Boock, U. and Njih Tabba, E. (2011) Traitement antibiotique: Évaluation à 12 mois de suivi après traitement d'une cohorte de patients au Cameroun. Réunion annuelle de l'OMS sur l'ulcère de Buruli, Genève, Suisse.

[39] Johnson, R.C., Nackers, F., Glynn, J.R., de Biurrun Bakedano, E., Zinsou, C., Aguiar, J., Tonglet, R. and Portaels, F. (2008) Association of HIV Infection and Mycobacterium ulcerans Disease in Benin. AIDS, 22, 901-903. https://doi.org/10.1097/QAD.0b013e3282f7690a

[40] Raghunathan, P.L., Withney, E.A., Asamia, K., et al. (2005) Risk Factors for Buruli Ulcer Disease (Mycobacterium ulcerans Infection): Results from Case-Control Study in Ghana. Clinical Infectious Diseases, 40, 1445-1453.

https://doi.org/10.1086/429623 
[41] Darie, H., Cautoclaud, A., Lajaunie, C. and Millet, P. (1994) Dermatological Aspects of AIDS in Western Africa. A Propos of 140 Cases. Bulletin De La Societe De Pathologie Exotique, 87, 176-180.

[42] WHO/HTM/NTD/IDM (2015) Management of Buruli Ulcer-HIV Coinfection: Technical Update.

[43] Dhople, A.M. and Namba, K. (2002) In Vitro Activity of Sitafloxacin (DU-6859a) Alone, or in Combination with Rifampicin, against Mycobacterium ulcerans. Journal of Antimicrobial Chemotherapy, 50, 727-729. https://doi.org/10.1093/jac/dkf218

[44] Phanzu, D. (2011) La confirmation de l'ulcère de Buruli dans le contexte de la décentralisation: Expérience du Projet Ulcère de Buruli de l'IME/Kimpese. Réunion annuelle de l'OMS sur l'ulcère de Buruli, Genève, Suisse.

[45] Mavinga Phanzu, D., Suykerbuyk, P., Saunderson, P., Ngwala Lukanu, P., Masamba Minuku, J.B., Imposo, D.B., et al. (2013) Burden of Mycobacterium ulcerans Disease (Buruli Ulcer) and the Underreporting Ratio in the Territory of Songololo, Democratic Republic of Congo. PLoS Neglected Tropical Diseases, 7, e2563. https://doi.org/10.1371/journal.pntd.0002563

[46] Porten, K., Sailor, K., Comte, E., Njikap, A., Sobry, A., Sihom, F., et al. (2009) Prevalence of Buruli Ulcer in Akonolinga Health District, Cameroon: Results of a Cross Sectional Survey. PLoS Neglected Tropical Diseases, 3, e466. https://doi.org/10.1371/journal.pntd.0000466

[47] Cassisa, V., Chauty, A., Ardant, M.F., Cottin, J., Koussemou, H., Johnson, C. and Marsollier, L. (2010) Rapport de la confirmation en laboratoire des cas de CDTUB Pobé (Bénin). Réunion annuelle de l'OMS sur l'ulcère de Buruli, Genève, Suisse.

[48] Ablordey, A. (2011) Intensifier la détection et le traitement précoces pour faire baisser la morbidité due à l'ulcère de Buruli dans le district d'Asante Akim North au Ghana. Réunion annuelle de l'OMS sur l'ulcère de Buruli, Genève, Suisse.

[49] Ayelo, G.A., Sopoh, G.E., Houezo, J.-G., Fiodessihoue, R., Affolabi, D., Dossou, A.D., et al. (2018) Improving Clinical and Epidemiological Predictors of Buruli Ulcer. PLoS Neglected Tropical Diseases, 12, e0006713. https://doi.org/10.1371/journal.pntd.0006713

[50] Yotsu, R.R., Murase, C., Sugawara, M., Suzuki, K., Nakanaga, K. and Ishii, N. (2015) Buruli Ulcer in Japan: Update. WHO Buruli Ulcer Meeting, Geneva, Switzerland.

[51] Lavender, C., Johnson, P. and Fyfe, J. (2010) Rapport sur la confirmation des cas d'UB au laboratoire, VIDRL, 2009. Réunion annuelle de l'OMS sur l'ulcère de Buruli, Genève, Suisse.

[52] Guimaraes-Peres, A., Portaels, F., De Rijk, P., Fissette, K., Pattyn, S., Van Vooren, J., et al. (1999) Comparison of Two PCRs for Detection of Mycobacterium ulcerans. Journal of Clinical Microbiology, 37, 206-208.

[53] Coulibaly, B., Coulibaly-Ngolo, M., Ekaza, E., Aka, N., Nguessan, K., Assande, J., et al. (2010) Mise en place e la culture in vitro de Mycobacterium ulcerans à partir d'échantillons cliniques versus recherche de BAAR et détection du génome bactérien à Abidjan, côte d'ivoire. Bulletin de la Société de Pathologie Exotique, 103, 2-7. https://doi.org/10.1007/s13149-009-0002-y

[54] Avokan, E. (2011) Apport de la PCR au diagnostic biologique de l'ulcère de Buruli au Bénin. Thèse de pharmacie, FSS Cotonou, No. 066, 66 p.

[55] Affolabi, D., Sanoussi, N., Vandelannoote, K., Odoun, M., Faihun, F., Anagonou, S., et al. (2012) Effects of Decontamination, DNA Extraction and Amplification Procedures on the Molecular Diagnosis of Mycobacterium Ulcerans Disease (Buruli 
Ulcer). Journal of clinical Microbiology, 50, 1195-1198.

https://doi.org/10.1128/JCM.05592-11

[56] Bretzel, G., Huber, K., Kobara, B., Beissner, M., Piten, E., et al. (2011) Laboratory Confirmation of Buruli Ulcer Disease in Togo, 2007-2010. PLoS Neglected Tropical Diseases, 5, e1228. https://doi.org/10.1371/journal.pntd.0001228

[57] Ngazoa-Kakou, S., Ekaza, E., Coulibaly, D., Aka, N., Coulibaly, B. and Dosso, M. (2010) Renforcement des Capacités pour le Diagnostic et la Surveillance de l'Ulcère de Buruli en Côte d'Ivoire: Mise au Point du Diagnostic PCR en Temps Réel. Réunion annuelle de l'OMS sur l'ulcère de Buruli, Genève, Suisse.

[58] Kamdem Simo, Y., Um Boock, A., Kabanda, C., Njih, E. and Eyangoh, S. (2010) Confirmation des cas d'ulcère de Buruli au Cameroun. Réunion annuelle de l'OMS sur l'ulcère de Buruli, Genève, Suisse. 